\title{
Biquadratic and ring exchange interactions in orthorhombic perovskite manganites
}

\author{
Natalya S. Fedorova, ${ }^{1, *}$ Claude Ederer, ${ }^{1}$ Nicola A. Spaldin, ${ }^{1}$ and Andrea Scaramucci ${ }^{1,2, \dagger}$ \\ ${ }^{1}$ Materials Theory, ETH Zürich, Wolfgang-Pauli-Strasse 27, CH-8093 Zürich, Switzerland \\ ${ }^{2}$ Laboratory for Developments and Methods, Paul Scherrer Institut, CH-5232 Villigen PSI, Switzerland \\ (Received 19 December 2014; revised manuscript received 27 February 2015; published 17 April 2015)
}

\begin{abstract}
We use $a b$ initio electronic structure calculations within the generalized gradient approximation (GGA+U) to density functional theory to determine the microscopic exchange interactions in the series of orthorhombic rare-earth manganites, o- $R \mathrm{MnO}_{3}$. Our motivation is to construct a model Hamiltonian (excluding effects due to spin-orbit coupling), which can provide an accurate description of the magnetism in these materials. First, we consider $\mathrm{TbMnO}_{3}$, which exhibits a spiral magnetic order at low temperatures. We map the exchange couplings in this compound onto a Heisenberg Hamiltonian and observe a clear deviation from the Heisenberg-like behavior. We consider first the coupling between magnetic and orbital degrees of freedom as a potential source of non-Heisenberg behavior in $\mathrm{TbMnO}_{3}$, but conclude that it does not explain the observed deviation. We find that higher order magnetic interactions (biquadratic and four-spin ring couplings) should be taken into account for a proper treatment of the magnetism in $\mathrm{TbMnO}_{3}$ as well as in the other representatives of the o- $R \mathrm{MnO}_{3}$ series with small radii of the $R$ cation.
\end{abstract}

\section{INTRODUCTION}

Perovskite manganites, $R \mathrm{MnO}_{3}\left(R^{3+}=\right.$ rare-earth cation), show a great variety of structural, magnetic, and electronic phases whose coexistence and interplay give rise to the large diversity of their physical properties. Orthorhombic $R \mathrm{MnO}_{3}$ (o- $R \mathrm{MnO}_{3}$ ) exhibiting frustrated magnetic orderings are of particular interest as they belong to the family of so-called magnetoelectric multiferroics-materials, where magnetic and ferroelectric orders are simultaneously presented [1-3]. Indeed, it has been shown experimentally, that the establishment of a spiral ordering of $\mathrm{Mn}^{3+}$ spins in $\mathrm{TbMnO}_{3}$ and $\mathrm{DyMnO}_{3}$ is accompanied by the appearance of a spontaneous electric polarization which can be manipulated by an applied magnetic field [4]. Recently a magnetically induced electric polarization was also observed in $\mathrm{o}-\mathrm{HoMnO}_{3}$ [5], which has an E-type antiferromagnetic order (E-AFM) [6]. Despite the fact that these effects occur at quite low temperatures, the understanding of their mechanisms is important for the fundamental physics of magnetoelectric phenomena and for potential development of multifunctional devices.

In this work we address the question of the origin of the frustrated magnetic orderings which cause the multiferroic properties in $\mathrm{o}-R \mathrm{MnO}_{3}$. According to experiment, the magnetic structure in the series of $0-R \mathrm{MnO}_{3}$ evolves from A-AFM to the spiral and then to the E-AFM state with decreasing radius of the $R$ cation, which favors the enhancement of orthorhombic distortion. This in turn changes the relative strength of nearest-neighbor $(\mathrm{NN})$ and further neighbor exchange interactions between $\mathrm{Mn}$ spins in these materials $[7,8]$. This evolution of the magnetic order is usually described within the framework of a Heisenberg model with competing NN and next-nearest-neighbor (NNN) exchanges. Indeed, qualitatively, this model gives the spiral as a ground state for a certain ratio between NN and NNN couplings

\footnotetext{
*natalya.fedorova@mat.ethz.ch

†andrea.scaramucci@psi.ch
}

$[3,9,10]$. However, as we will show in details in Sec. II B, application of this model for quantitative description of the exchanges in o- $R \mathrm{MnO}_{3}$ gives contradictory results. Moreover, it was shown recently, that the E-AFM state cannot be obtained from the Heisenberg Hamiltonian [11].

Here we present the results of our studies of the microscopic magnetic couplings in the series of $0-R \mathrm{MnO}_{3}$ using firstprinciples electronic structure calculations with the goal of finding a model Hamiltonian which can accurately describe the magnetism in these materials. We restrict ourselves to the analysis of the exchange interactions which are independent of spin-orbit coupling, thus single-ion anisotropy (SIA) and Dzyaloshinskii-Moriya interaction (DMI) are excluded in this work. First we consider $\mathrm{TbMnO}_{3}$ with spiral spin ordering. We map the exchanges in this compound onto the Heisenberg model and find a clear deviation from Heisenberg-like behavior. We investigate the extent to which this deviation originates from the presence of the orbital ordering in $\mathrm{TbMnO}_{3}$ and show that the coupling between magnetic and orbital degrees of freedom cannot provide the observed deviation. Then we explore the effect of exchange couplings of higher orders than the bilinear exchange (biquadratic and four-spin ring couplings), which are usually neglected. We demonstrate that the higher order contributions are significant in $\mathrm{TbMnO}_{3}$ and other o- $R \mathrm{MnO}_{3}$ with small radii of the $R$ cation (independently of the choice of the DFT+U parameters, which are used in the calculations) and that they have to be included in the model Hamiltonian for an accurate description of the magnetic properties of orthorhombic manganites.

This article is organized as follows: In Sec. II we describe the crystal structure, the orbital ordering and its relation to the magnetic properties in $0-\mathrm{RnO}_{3}$, and explain the motivation of our research. Here we also introduce the methods which we use in our calculations and specify the computational details. In Sec. III we calculate the microscopic exchange couplings in $\mathrm{TbMnO}_{3}$ and show that they cannot be described by the Heisenberg Hamiltonian. In Sec. IV we discuss the possible sources of the non-Heisenberg behavior in $\mathrm{TbMnO}_{3}$; in particular, we investigate the effects of orbital 
(a)

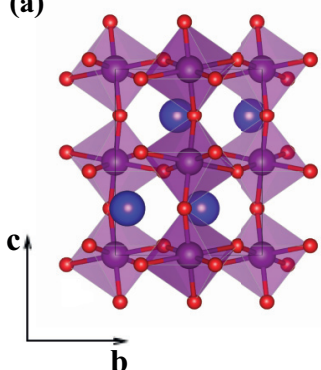

(c)

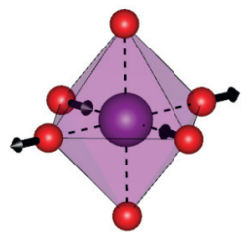

(b)

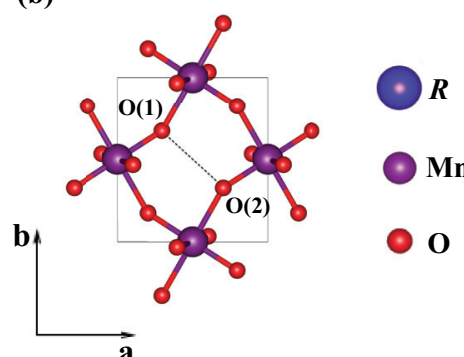

(d)

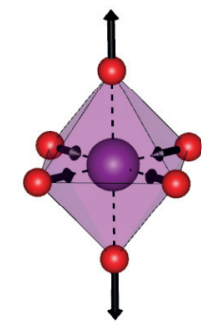

FIG. 1. (Color online) Crystal structure of o- $R \mathrm{MnO}_{3}$ : (a) side view; (b) top view ( $R$ ions are not shown). (c) and (d) Representation of the normal modes of Jahn-Teller distortion $Q_{2}$ and $Q_{3}$, respectively.

ordering, structural distortions, and higher order exchange couplings. In Sec. V we analyze how the choice of DFT+U parameters, specifically the on-site Coulomb repulsion $U$ and on-site exchange interaction $J_{H}$, affects the resulting values of microscopic exchange interactions in $\mathrm{TbMnO}_{3}$. In Sec. VI we extend our analysis on the other representatives of the o- $R \mathrm{MnO}_{3}$ series, namely, $\mathrm{PrMnO}_{3}$ and $\mathrm{LuMnO}_{3}$. Finally, in Sec. VII we summarize our work and give a conclusion.

\section{MOTIVATION, THEORETICAL BACKGROUND, AND METHODS}

\section{A. Jahn-Teller and $\mathrm{GdFeO}_{3}$-type distortions in $0-\mathrm{RMnO}_{3}$}

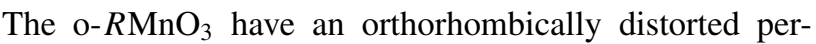
ovskite structure (see Fig. 1) with space group Pbnm (\#62) and 20 atoms per unit cell [4,12-14]. The deviation from the perfect cubic perovskite structure includes the Jahn-Teller distortion of the $\mathrm{MnO}_{6}$ octahedra [15], their cooperative tiltings [16] (the so-called $\mathrm{GdFeO}_{3}$-type, GFO, distortion) and small antiferroelectric displacements of $R$ cations from their ideal positions [17]. While the latter structural distortion has been shown to influence the ferroelectric properties, its effect on the magnetism is negligible and we do not consider it in this work.

In $\mathrm{o}-\mathrm{RMnO}_{3}$ each $\mathrm{Mn}^{3+}$ ion resides in the middle of an oxygen octahedron with four electrons in $3 d$ levels. The crystal field of the perfect octahedron splits the fivefold degenerate $d$ levels into triply degenerate $t_{2 g}$ lower-energy levels and doubly degenerate $e_{g}$ levels with higher energy. Electrons occupy the orbitals according to Hund's rules and the Pauli principle, which leads to full occupation of the spin majority $t_{2 g}$ states and single occupation of the spin majority $e_{g}$ states. Lowering of the symmetry of the crystal field due to the Jahn-Teller effect [18] lifts the degeneracy of the $e_{g}$ electronic state and favors the occupation of a certain orbital which can be represented as

a superposition of $d_{z^{2}}$ and $d_{x^{2}-y^{2}}$ states [19]:

$$
|\psi\rangle=\cos \left(\frac{\theta}{2}\right)\left|d_{z^{2}}\right\rangle+\sin \left(\frac{\theta}{2}\right)\left|d_{x^{2}-y^{2}}\right\rangle .
$$

The state $|\psi\rangle$ is uniquely defined by the angle $\theta$ which is called the orbital mixing angle. The corresponding distortion of the octahedron can be written as a linear combination of two normal Jahn-Teller modes $Q_{2}$ and $Q_{3}$ [15,20] [Figs. 1(c) and $1(\mathrm{~d})$, respectively]:

$$
Q=Q_{3} \cos \varphi+Q_{2} \sin \varphi
$$

The value of $\varphi$ can be estimated with the simple formula,

$$
\varphi=\arctan \left(\frac{Q_{2}}{Q_{3}}\right)=\arctan \left(\frac{\sqrt{3}(l-s)}{2 m-l-s}\right),
$$

where $l, m$, and $s$ are the lengths of the long, medium and short $\mathrm{Mn}-\mathrm{O}$ bonds in the octahedron [21]. The ground-state value of $\theta$ is determined by the balance between the energy gain due to the orbital-lattice interaction and the elastic energy cost [22]. For a single octahedron this occurs at $\varphi=\theta$.

Since the oxygen octahedra are interconnected, their distortions and, therefore, the occupied orbital states on neighboring $\mathrm{Mn}$ ions are not independent. Below a certain temperature, this leads to a long-range orbital ordering with the orbital mixing angles for two neighboring Mn sites $i$ and $j$ in the $a b$ plane related by $\theta_{i}=-\theta_{j}$ (antiferro-orbital orientation). For nearest neighbors along the $c$ direction they are equal (ferro-orbital orientation).

The GFO distortion is characterized by almost rigid cooperative rotations of the $\mathrm{MnO}_{6}$ octahedra, which result in the reduction of $\mathrm{Mn}-\mathrm{O}-\mathrm{Mn}$ bond angles and $\mathrm{O}(1)-\mathrm{O}(2)$ distances [see Fig. 1(b)]. In the series of $\mathrm{o}-R \mathrm{MnO}_{3}$ this distortion increases with decreasing radius of the $R$ cation from La to Lu.

\section{B. Frustrated magnetism in $0-\mathrm{RMnO}_{3}$}

The combination of Jahn-Teller and GFO distortions in $\mathrm{O}-\mathrm{R} \mathrm{MnO}_{3}$ determines their magnetic properties [23]. According to the Goodenough-Kanamori-Anderson rules [24-26], the presence of the orbital ordering of the type which was described in the previous section favors FM exchange coupling between neighboring $\mathrm{Mn}$ spins in the $a b$ planes and AFM coupling along the crystallographic $c$ direction. This promotes the establishment of A-AFM ordering for $R=\mathrm{La} \ldots \mathrm{Gd}$. However, further decreasing the size of $R$ cation in the series of $\mathrm{o}-R \mathrm{MnO}_{3}$ (and, therefore, increasing the GFO distortion) causes the transition to the spiral ( $R=\mathrm{Tb}$, Dy) and then E-AFM states ( $R=$ Ho . . Lu). One can consider the change in the relative strength of FM NN and AFM NNN couplings in the $a b$ plane as an origin of this transition. Indeed, increasing GFO distortion decreases NN exchange as it strongly depends on the Mn-O-Mn bond angles. On the other hand, it enhances the AFM exchange between NNNs along the $b$ axis through the path $\mathrm{Mn}-\mathrm{O}(1)-\mathrm{O}(2)-\mathrm{Mn}$ due to the reduction of $\mathrm{O}(1)-\mathrm{O}(2)$ distances [see Fig. 1(b)]. This strong AFM NNN exchange causes magnetic frustration [8].

The simplest microscopic model which is often used to discuss this evolution of the magnetic order is the Heisenberg 
model:

$$
H_{\mathrm{Heis}}=\sum_{\langle i, j\rangle} J_{i j} \mathbf{S}_{i} \cdot \mathbf{S}_{j},
$$

where $J_{i j}$ indicates exchange interactions between spins $\mathbf{S}_{i}$ and $\mathbf{S}_{j}$. As exchange interactions are short ranged, usually only the couplings between first- and second-nearest neighbors are taken into account [3]. This model qualitatively explains the establishment of spiral magnetic ordering. Indeed, for o$R \mathrm{MnO}_{3}$ the ratio,

$$
\frac{J_{b}}{\left|J_{a b}\right|}>\frac{1}{2}
$$

for $a b$ plane FM NN exchange $J_{a b}$ and AFM NNN exchange $J_{b}$ along the axis $b$ gives a spiral (with a propagation vector along the $b$ axis) as a magnetic ground state [27]. However, the source of E-AFM ordering is still under debate. For example, Kimura et al. [8] stated that the two-dimensional Heisenberg model with FM NN and certain competing AFM NNN couplings in the $a b$ plane can give E-AFM ordering, whereas Kaplan and Mahanti [11] demonstrated that such a state cannot occur in this model. Later it was shown for the example of a onedimensional spin chain, that addition of a biquadratic nearestneighbor exchange interaction of the form,

$$
H_{b q}=\sum_{\langle k, l\rangle} j_{k l}\left(\mathbf{S}_{k} \cdot \mathbf{S}_{l}\right)^{2},
$$

to the Heisenberg Hamiltonian gives rise to E-AFM order [10]. Then the importance of biquadratic coupling in the establishment of an E-AFM state was also confirmed for the two-dimensional case [28]. In turn, Solovyev [29] claimed that it is crucial to consider the exchange interaction between the third nearest neighbors in the $a b$ planes to stabilize the E-AFM state.

Aside from the disagreement on the source of the EAFM order, the application of the Heisenberg model for a quantitative description of the magnetism in $\mathrm{o}-\mathrm{RMnO}$ gives ambiguous results. Assuming that the magnetism in $\mathrm{o}-R \mathrm{MnO}_{3}$ is fully described by the Heisenberg Hamiltonian and considering only the couplings between NN spins, the total energy can be written as

$$
E=\sum_{\langle i, j\rangle} J_{i j} \mathbf{S}_{i} \cdot \mathbf{S}_{j}+E_{0},
$$

where $E_{0}$ includes all other (nonmagnetic) interactions. Therefore, one can see that the difference in the total energies of the unit cell of $\mathrm{o}-R \mathrm{MnO}_{3}$ with A-AFM and G-AFM orientations [see Figs. 2(a) and 2(b), respectively] of $\mathrm{Mn}^{3+}$ spins $\left(\Delta E_{\mathrm{AG}}\right)$ defines the value of exchange coupling $J_{a b}$. Moreover, this value should be the same as that given by the difference in the energies of FM and C-AFM [Figs. 2(d) and 2(c), respectively] states $\left(\Delta E_{\mathrm{FC}}\right)$. Similarly, the NN exchange $J_{c}$ along the $c$ axis can be extracted from the following energy differences: $\Delta E_{\mathrm{FA}}=E(\mathrm{FM})-E(\mathrm{~A}-\mathrm{AFM})$ and $\Delta E_{\mathrm{CG}}=E(\mathrm{C}-\mathrm{AFM})-E(\mathrm{G}-\mathrm{AFM})$ and the obtained values should be the same for these two cases. The energies of FM, A-AFM, C-AFM, and G-AFM states have been calculated by several groups for the series of o- $R \mathrm{MnO}_{3}$ applying different theoretical approaches [29-31]. Using the published values (a)
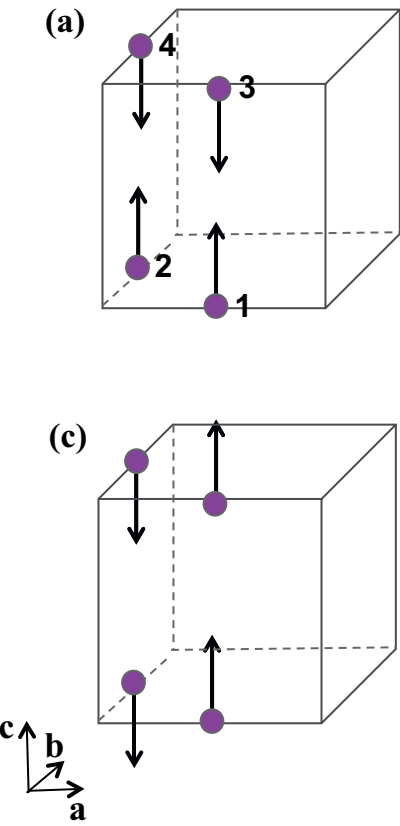

(b)

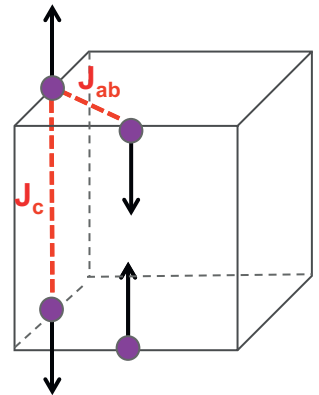

(d)

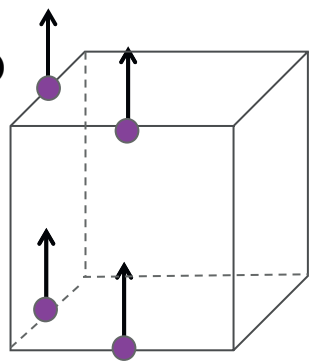

FIG. 2. (Color online) Collinear magnetic orderings (in WollanKoehler notation [6]) of the Mn spins within the perovskite unit cell: (a) A-AFM, (b) G-AFM, (c) C-AFM, and (d) FM. $J_{c}$ and $J_{a b}$ indicate the NN exchange couplings along the $c$ axis and within the $a b$ planes, respectively.

of these energies, we calculate for each case $\Delta E_{\mathrm{AG}}$ and $\Delta E_{\mathrm{FC}}$, which define $J_{a b}$ and should give the same results. However, we find that the obtained values $\Delta E$ are significantly different. The same is found for $J_{c}$, where $\Delta E_{\mathrm{FA}}$ and $\Delta E_{\mathrm{CG}}$ give in some cases even different signs. As an example, we present in Table I the values of $J_{a b}$ and $J_{c}$ in $\mathrm{LaMnO}_{3}$ obtained using the generalized gradient approximation in the form of Perdew, Burke, and Ernzerhof (GGA-PBE)[30], Hartree-Fock approximation (HFA) [29], and GGA with the Perdew-Wang-91 functional (GGA-PW91) [31]. We would like to point out, that we do not compare the values of $J_{c}$ and $J_{a b}$ obtained with different approximations and presented in different rows of Table I. For each approximation we compare two values of $J_{a b}\left(J_{c}\right)$, which were obtained using $\Delta E_{\mathrm{AG}}$ and $\Delta E_{\mathrm{FC}}\left(\Delta E_{\mathrm{FA}}\right.$ and $\left.\Delta E_{\mathrm{CG}}\right)$ and, in principle, should give very similar values. Table I also demonstrates that the inconsistencies in the values of exchanges are not related to the choice of the exchange-correlation potential. It should be

TABLE I. The exchange parameters $J_{a b}$ and $J_{c}$ (in $\mathrm{meV}$ ) calculated with different theoretical approaches for $\mathrm{LaMnO}_{3}$ using normalized values of spins $\left|\mathbf{S}_{i}\right|=\left|\mathbf{S}_{j}\right|=1$.

\begin{tabular}{lccrrr}
\hline \hline & \multicolumn{2}{c}{$J_{a b}$} & & \multicolumn{2}{c}{$J_{c}$} \\
\cline { 2 - 3 } \cline { 5 - 6 } Method & $\Delta E_{\mathrm{AG}}$ & $\Delta E_{\mathrm{FC}}$ & & $\Delta E_{\mathrm{FA}}$ & $\Delta E_{\mathrm{CG}}$ \\
\hline GGA-PBE [30] & -27.7 & -22.5 & & 0.5 & -10.0 \\
HFA [29] & -4.75 & -1.25 & & 10.0 & 3.0 \\
GGA-PW91 [31] & -18.0 & -14.6 & & 5.0 & -1.75 \\
\hline \hline
\end{tabular}


noted that the addition of the biquadratic term [Eq. (6)] in the Hamiltonian cannot explain these results as it cancels out in each energy difference.

These inconsistencies have not been addressed in the literature and require further investigation. On one hand, they could arise from the presence of strong NNN couplings. On the other hand, they could point to the presence of other significant couplings beyond the Heisenberg Hamiltonian, which have to be taken into account for a proper theoretical analysis of the magnetism in o- $R \mathrm{MnO}_{3}$.

\section{Mapping of DFT onto the Heisenberg model}

In order to examine the relevance of the Heisenberg model for $\mathrm{o}-R \mathrm{MnO}_{3}$ and to clarify the inconsistencies in the previous theoretical results, we perform a thorough analysis of the microscopic exchange couplings by mapping the results of density functional theory (DFT) [32,33] calculations onto the Heisenberg Hamiltonian. For that purpose we use two approaches, described below in this section and based on certain modifications of the initial magnetic states. In this context we point out that in the DFT calculations periodic boundary conditions are applied and a variation of a state of a Mn spin on one site leads to the same variation of Mn spin states on all periodically equivalent sites.

\section{Calculations with collinear spin configurations}

The first approach, described in detail in Refs. [34,35], is based on calculations of the total energy of the system with collinear spin alignment when the spin states on two sites (let us denote them as 1 and 2) within the given unit cell are modified. If the magnetism in the system is fully described by the Heisenberg Hamiltonian, its total energy can be written as follows:

$$
E=n J_{12} \mathbf{S}_{1} \cdot \mathbf{S}_{2}+\mathbf{S}_{1} \cdot \mathbf{h}_{1}+\mathbf{S}_{2} \cdot \mathbf{h}_{2}+E_{\text {all }}+E_{0},
$$

where $n$ is the number of equivalent bonds with exchange coupling $J_{12}$, which connect ions 1 and $2, \mathbf{h}_{1}=\sum_{i \neq 1,2} J_{1 i} \mathbf{S}_{i}$, $\mathbf{h}_{2}=\sum_{i \neq 1,2} J_{2 i} \mathbf{S}_{i}$, and $E_{\text {all }}=\sum_{i, j \neq 1,2} J_{i j} \mathbf{S}_{i} \cdot \mathbf{S}_{j} ; E_{0}$ contains all nonmagnetic energy contributions. Taking into account periodic boundary conditions, one should consider the first term in Eq. (8) as the Heisenberg exchange interactions between spins of magnetic sublattices 1 and 2 [spin on the site 1 (or 2) and on all translationally equivalent sites], the second (third) term as the coupling of the spins of the sublattice 1 (2) with all other sublattices except 2 (1), and $E_{\text {all }}$ as the exchange couplings between all magnetic sublattices apart from 1 and 2. The number of sublattices is determined by the size of chosen unit cell. Four different collinear configurations of the spins 1 and 2 are possible-up-up, up-down, down-up, and down-down and the corresponding energies can be calculated using DFT. Then, the exchange interaction between sublattices 1 and 2 can be found using the formula,

$$
J_{12}=\frac{E_{\uparrow \uparrow}+E_{\downarrow \downarrow}-E_{\uparrow \downarrow}-E_{\downarrow \uparrow}}{4 n S^{2}} .
$$

For a more direct comparison with other materials we prefer not to normalize our reported values of $J_{i}$ by $S^{2}$ (thus we set $S=1$ for Mn). Substituting in this expression the energies $E_{\uparrow \uparrow}, E_{\downarrow \downarrow}, E_{\uparrow \downarrow}$, and $E_{\downarrow \uparrow}$ using Eq. (8), one sees that all terms, except those describing the exchange interaction between sublattices 1 and 2, cancel out. As a result, the parameter $J_{12}$ does not contain contributions from couplings between the spins of sublattices 1 and 2 and those of other sublattices and, therefore, should not depend on the orientation of spins that do not belong to the sublattices 1 and 2 .

\section{Noncollinear calculations}

This approach is based on the calculation of the total energy of the system when some spins are rotated away from an initial collinear state [36] and can be illustrated by the example of the unit cell with four magnetic ions. We consider A-AFM ordering for spins in the unit cell as a starting point and rotate the spins of ions 2 and 4 [see Fig. 2(a)] by an angle $\alpha$ keeping them antiparallel to each other until we reach G-AFM ordering [Fig. 2(b)].

The energy of the system as a function of $\alpha$ within the Heisenberg model can be written as

$$
E(\alpha)=-4 J_{c} S^{2}+8 J_{a b} S^{2} \cos \alpha+E_{0},
$$

and can be calculated using DFT. The resulting curve should fit the form,

$$
f(\alpha)=A_{1}+B_{1} \cos \alpha
$$

if the Heisenberg model provides an accurate description (independently of the number of considered exchange couplings as periodic boundary conditions are applied) and the fitting parameter $B_{1}$ should define the exchange coupling constant $J_{a b}=B_{1} / 8 S^{2} . J_{c}$ can be extracted similarly by rotating spins on sites 3 and 4 from G-AFM to C-AFM ordering [from Fig. 2(b) to 2(c), respectively].

\section{Computational details}

We perform spin-polarized electronic structure calculations using the Vienna $A b$ initio Simulation Package (VASP) [37] within the projector-augmented plane wave (PAW) method of DFT. We use the GGA+U approximation for the exchangecorrelation potential (in the form of Perdew, Burke, and Ernzerhof [38]). Unless otherwise stated, we apply an on-site Coulomb repulsion of $U=2 \mathrm{eV}$ to the $\mathrm{Mn} d$ states as implemented in Liechtenstein's scheme [39] and set the parameter of the effective on-site exchange (Hund's rule) interaction $J_{H}=$ $0 \mathrm{eV}$. This is equivalent to using $U_{\text {eff }}=U-J_{H}=2 \mathrm{eV}$ in Dudarev's scheme [40]. We do not include spin-orbit coupling in our calculations, thus neither SIA nor DMI (nor any other magnetic interactions with relativistic origin) can contribute to the resulting total energies. To eliminate the effects from the ordering of the f-electron moments of rare-earth ions, we use pseudopotentials for $R$ with the $f$ states frozen in the core. The value of the energy cutoff is set to $600 \mathrm{eV}$. In all calculations we fix the crystal structures to the experimental ones $[12,41,42]$ to isolate any contributions from spin-lattice coupling. To construct the set of projected Wannier functions [43] we use the WANNIER90 [44] code and the VASP2WANNIER90 interface [45]. 


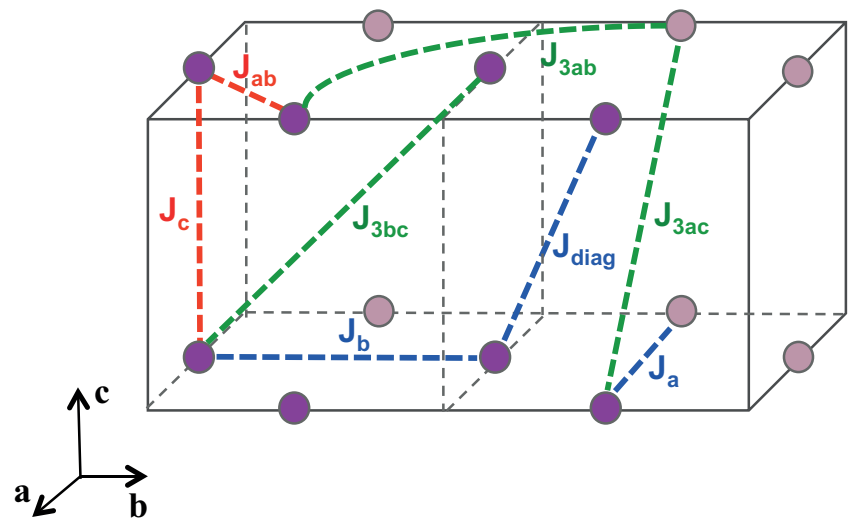

FIG. 3. (Color online) Heisenberg interactions in $\mathrm{TbMnO}_{3}$, which are considered in this work. Mn atoms within the 40-atom supercell are highlighted with dark purple. Light purple circles indicate $\mathrm{Mn}$ ions which belong to the neighboring supercells. NN exchanges are indicated in red, second NN in blue, and third NN in green.

\section{DEVIATION FROM HEISENBERG MODEL}

We start with the analysis of the microscopic exchange couplings in the most studied multiferroic orthorhombic perovskite compound $\mathrm{TbMnO}_{3}[46,47]$. We initially assume that the magnetism in this material is fully described by the Heisenberg Hamiltonian [Eq. (4)] and limit ourselves to consideration of the exchange couplings up to third NN within the $a b, b c$, and $a c$ planes. In our notation $J_{c}$ and $J_{a b}$ are the NN exchanges along the $c$ axis and in the $a b$ plane (see Fig. 3) respectively; $J_{a}$ corresponds to the second NN exchange along the $a$ direction, $J_{b}$-along the $b$ axis and $J_{\text {diag }}$ couples second $\mathrm{NN}$ in adjacent $a b$ planes; $J_{3 a b}, J_{3 b c}$, and $J_{3 a c}$ are exchanges between third NN in the $a b, b c$, and $a c$ planes, respectively. We extract these parameters applying the method described in Sec. II C 1. For this purpose we consider an 80 atom supercell (the orthorhombic unit cell [12] is doubled in the $a$ and $b$ directions) and a $\Gamma$-centered $3 \times 3 \times 5 k$-point mesh. For each $J$ we choose the corresponding spin pair in the supercell and calculate the total energies of the system for the four possible orientations of spins in this pair (up-up, up-down, down-up, down-down). We keep the rest of Mn spins fixed first in the FM state (FM case) and then in the A-AFM state (A-AFM case). Note that for $J_{\text {diag }}$ and $J_{3 a b}$ there are two types of spin pairs which are not related by symmetry operations of the Pbnm space group and we calculate these coupling constants for both of them. The obtained values of $J$ are presented in Table II. We

TABLE II. Values of $\mathrm{NN}$ and NNN exchange interactions (in $\mathrm{meV}$ ) in $\mathrm{TbMnO}_{3}$ calculated for FM and A-AFM cases using $U=$ $2 \mathrm{eV}$ and $J_{H}=0 \mathrm{eV} . d(\mathrm{Mn}-\mathrm{Mn})$ indicates the $\mathrm{Mn}-\mathrm{Mn}$ distance (in $\AA$ ) corresponding to each $J_{i}$.

\begin{tabular}{lcccccccc}
\hline \hline & $J_{c}$ & $J_{a b}$ & $J_{a}$ & $J_{\text {diag }}$ & $J_{b}$ & $J_{3 a b}$ & $J_{3 b c}$ & \multicolumn{1}{c}{$J_{3 a c}$} \\
$d(\mathrm{Mn}-\mathrm{Mn})$ & 3.701 & 3.940 & 5.293 & 5.406 & 5.838 & 7.881 & 6.913 & 6.459 \\
\hline FM & 3.68 & -4.62 & -0.06 & 0.97 & 1.10 & 1.11 & 0.11 & -0.02 \\
A-AFM & -0.85 & -5.16 & -0.32 & -0.10 & 0.68 & 1.26 & 0.007 & 0.03 \\
\hline \hline
\end{tabular}

(a)

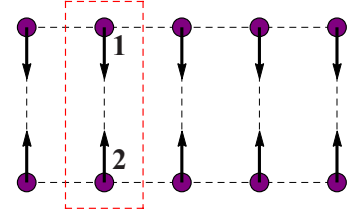

(c)

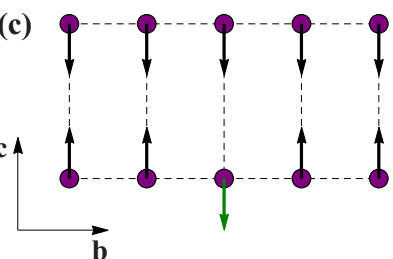

(b)

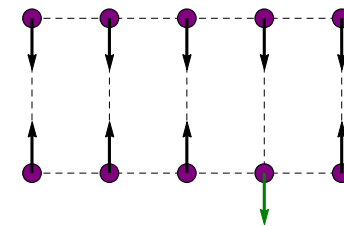

(d)

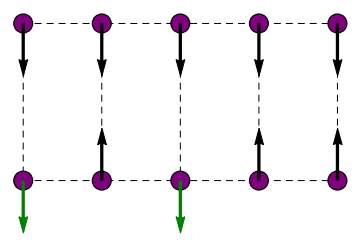

FIG. 4. (Color online) Forty atom supercell of $\mathrm{TbMnO}_{3}$ (side view) with magnetic orders which were used to calculate the exchange parameter $J_{c}$. Tb and $\mathrm{O}$ ions are not shown.

find that for our chosen values of $U$ and $J_{H}, J_{b}$ is rather weak relative to $J_{a b}$ for both (FM and A-AFM) cases and according to Eq. (5) cannot produce the spiral state in $\mathrm{TbMnO}_{3}$. The other possible source of frustration could be the AFM coupling $J_{3 a b}$ which is stronger even than the second NN in-plane couplings $J_{a}$ and $J_{b}$. The importance of $J_{3 a b}$ was already pointed out in Ref. [29], where strong or weak $J_{3 a b}$ couplings were proposed depending on the relative orientation of the occupied $d$ orbitals on the interacting sites (two inequivalent-by-symmetry spin pairs). However, we obtain the same value of $J_{3 a b}$ for both orbital orientations. Similarly, we obtain identical values of $J_{\text {diag }}$ for the two symmetry inequivalent spin pairs. Therefore we introduce only one coupling constant for both $J_{\text {diag }}$ and $J_{3 a b}$. The third NN couplings, $J_{3 b c}$ and $J_{3 a c}$, were found to be weak in comparison with the other couplings and they will not be considered further in this work.

The key result of these calculations is that the values of the exchanges, especially $J_{c}$, have different magnitudes and in some cases even different signs for FM and A-AFM cases while within the Heisenberg description they should be equal (or at least very similar). We point out that such an inconsistency cannot arise from further neighbor exchange couplings. Indeed, as we discussed in Sec. II C 1, all further neighbor contributions cancel each other in Eq. (9).

To double check this result and to determine its origin, we extract $J_{c}$, which shows the largest inconsistency, with the same method using a 40-atom supercell (20-atom unit cell doubled along the $b$ axis, $7 \times 4 \times 5 \Gamma$-centered $k$-point mesh). We calculate the total energies switching the direction of spins 1 and 2, but now the rest of the spins are kept in the states shown in Fig. 4. The new calculated $J_{c}$ values are presented in Table III. One can see that the $J_{c}$ value obtained using state (a) (which is A-AFM order) is in agreement with the $J_{c}$ value which was found using the 80 atom supercell and starting from the same magnetic state. Interestingly, the values of $J_{c}$ are similar for the states (a) and (b), where the closest surroundings of spins 1 and 2 are identical. In turn, if the states differ by the direction of one spin in the nearest neighborhood of the considered spin pair [such as between states (a) and (c) or (c) and (d)], $J_{c}$ changes by approximately the same amount (in average by $1.85 \mathrm{meV}$ ). This suggests the presence of strong couplings beyond the Heisenberg Hamiltonian which 
TABLE III. The values of the exchange coupling constant $J_{c}$ (in $\mathrm{meV}$ ) in $\mathrm{TbMnO}_{3}$ calculated using the magnetic states shown in Fig. 4. $E_{4 s p}$ indicates the contributions to the values of $J_{c}$ from four-spin ring exchange $K$ between Mn spins confined in adjacent $a b$ planes.

\begin{tabular}{lcccc}
\hline \hline & (a) & (b) & (c) & (d) \\
\hline$J_{c}$ & -0.88 & -0.68 & 0.92 & 2.84 \\
$E_{4 s p}$ & $-4 K$ & $-4 K$ & 0 & $4 K$ \\
\hline \hline
\end{tabular}

involve in some way the magnetic interactions between the nearest-neighboring Mn spins.

Another check can be done by the method described in Sec. II C 2. To apply this method we consider a 20-atom unit cell and a $5 \times 5 \times 3 k$-point mesh. We rotate the spins on sites 2 and 4 from A-AFM to G-AFM ordering (see Fig. 2) and calculate the energies $E$ of the system (relative to the energy of A-AFM order) for several values of spin rotation angle $\alpha$ between $0^{\circ}$ and $180^{\circ} . E(\alpha)$ and its fitting to $f(\alpha)$ [Eq. (11)] are presented in Fig. 5 (black dots and blue line, respectively). One can see that $E(\alpha)$ shows clear deviation from the cosinusoidal behavior predicted by the Heisenberg model.

These results lead us to the conclusion that the Heisenberg Hamiltonian in its usual form is not able to accurately describe the magnetism in $\mathrm{TbMnO}_{3}$ and more couplings have to be taken into consideration.

\section{ORIGIN OF NON-HEISENBERG BEHAVIOR}

\section{A. Orbital ordering}

First, we investigate whether the observed non-Heisenberg behavior can originate from the presence of the orbital ordering in $\mathrm{TbMnO}_{3}$. Indeed, as was already described in Sec. II B, the magnetic and orbital orderings are related as far as the coupling of spins on neighboring $\mathrm{Mn}^{3+}$ ions is determined by the occupation of their particular orbitals through the superexchange mechanism. To take this behavior into account,

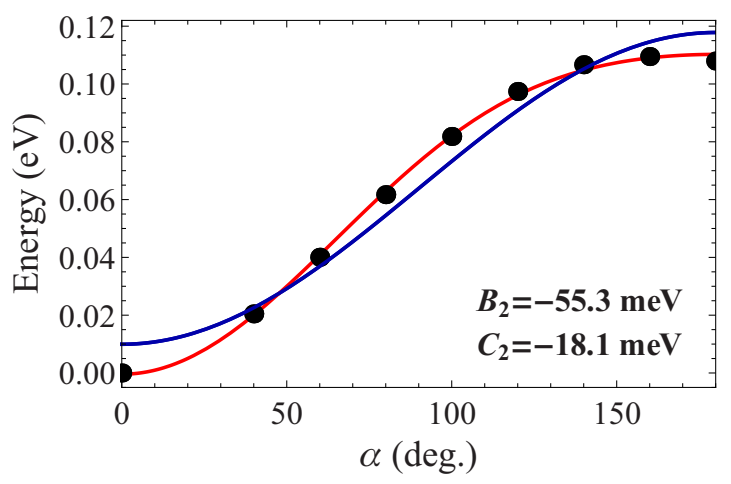

FIG. 5. (Color online) Dependence of the energy $E$ (relative to the energy of A-AFM order) of $\mathrm{TbMnO}_{3}$ on the rotation angle $\alpha$ of spins from the A-AFM to G-AFM state calculated using $U=2 \mathrm{eV}$ and $J_{H}=0 \mathrm{eV}$. The results of DFT calculations are shown by dots and the fitting to the Heisenberg model [Eq. (11)] by the blue line. The red line indicates the fitting to a Hamiltonian which includes bilinear and higher order exchange couplings [Eq. (15)], $B_{2}$ and $C_{2}$ are the fitting parameters, which define the sizes of the bilinear and higher order terms, respectively.

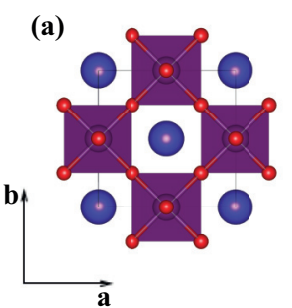

(b)

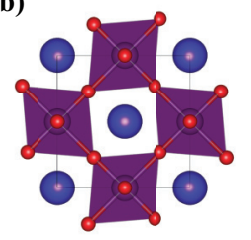

(c)

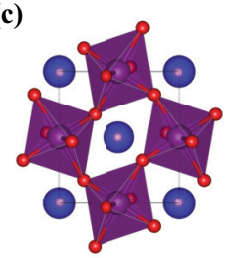

FIG. 6. (Color online) (a) Ideal cubic perovskite structure; (b) purely JT distorted structure (tetragonal); (c) fully JT+GFO distorted structure (orthorhombic) of $\mathrm{TbMnO}_{3}$.

the ordinary superexchange was generalized for the case of systems with orbital degeneracy by Kugel and Khomskii [19]. They introduced a model Hamiltonian, in which, besides the Heisenberg exchange, they included terms describing orbitalorbital and orbital-spin couplings. The latter gives the change in the orbital ordering with variation of the spin alignment (or vice versa) and, if it is large enough, can explain the different values of exchange obtained for A-AFM and FM cases as well as the deviation from Heisenberg behavior observed in noncollinear calculations.

The occupied $e_{g}$ orbital $|\psi\rangle$ for each $\mathrm{Mn}^{3+}$ ion is uniquely defined in terms of the orbital mixing angle $\theta$ by Eq. (1). To extract $\theta$ for $\mathrm{TbMnO}_{3}$, so as to trace its evolution with structural distortions and to estimate the strength of coupling between orbitals and spins we perform the following analysis: We construct a perfect cubic perovskite structure for $\mathrm{TbMnO}_{3}$ using a 20-atom unit cell and keeping the volume of each $\mathrm{MnO}_{6}$ octahedron equal to the experimental one [see Fig. 6(a)]. Then we start to apply the JT modes $\mathrm{Q}_{2}$ and $\mathrm{Q}_{3}$ (without GFO distortion) in such a way that $Q_{i \text {,applied }}=a Q_{i \text {,exp }}$ [thus the angles $\varphi$, which are defined by Eq. (3), are equal for all values of $a$ ]. $a$ is varied from 0 to 1 and $Q_{i, \exp }$ corresponds to the structure with the full JT distortion [Fig. 6(b)]. To reach a more transparent description of the orbital ordering, we use a representation in terms of Wannier functions [48] (WF), which, unlike Bloch functions, are localized in space and have minimal overlap with the surrounding orbitals. To construct a set of $e_{g}$ like WFs, we proceed similarly to Refs. [43,49]. We calculate the Bloch functions within GGA+U (using the structures corresponding to different $a$ ), and for each structure we define an energy window, in which $e_{g}$ bands are located, based on projected densities of states and band structures. Then, using the VASP2WANNIER90 interface and WANNIER90 code, we construct four WFs via projection of atomiclike $\left|d_{z^{2}}\right\rangle$ and $\left|d_{x^{2}-y^{2}}\right\rangle$ orbitals centered on two Mn sites (1 and 2) on the majority spin Bloch bands within the chosen energy window. Then we calculate the occupation matrices in the basis of these WFs for several values of $a$ and two types of ordering of the Mn magnetic moments (A- and G-AFM). Solving the eigenvalue problem for these matrices, we find $\left|\psi_{i}\right\rangle(i=1,2)$ and, thus, $\theta_{i}$. We plot $\theta_{1}$ as a function of $a$ starting from $a=0.4$ (see Fig. 7, left half of the graph), since smaller amplitudes of JT distortion give a metallic ground state. $\theta_{2}$ has the same values as $\theta_{1}$, but the opposite sign.

As we expected, the calculated $\theta$ are different for A- and G-AFM orderings. With increasing JT distortion from $40 \%$ to $100 \%$ the mixing angles for both AFM orders change by 


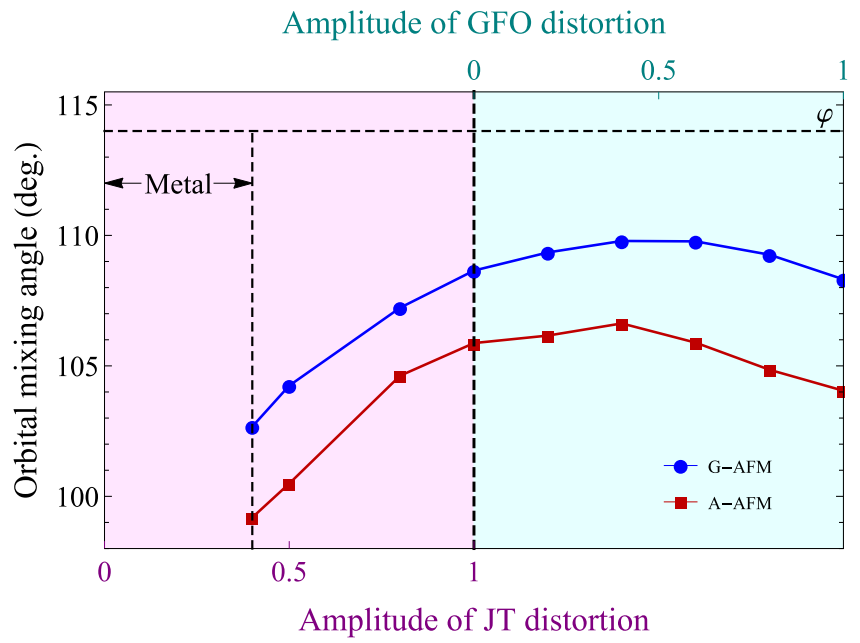

FIG. 7. (Color online) Orbital mixing angle as a function of the amplitudes of JT and $\mathrm{GdFeO}_{3}$-type distortions for G-AFM and A-AFM magnetic orderings in $\mathrm{TbMnO}_{3}$. In the part of the graph highlighted with violet (cyan), only the amplitude of the JT (GFO) distortion is varied. $\varphi$ is determined using Eq. (3).

approximately $6^{\circ}$ and tend to the value of $\varphi \approx 114^{\circ}$ which is imposed by the structure $[\varphi$ is calculated using Eq. (3) and experimental lengths of long, medium, and short bonds in Mn-O octahedra]. It is important, that the difference in the orbital mixing angles $\Delta \theta$ between A- and G-AFM orderings (in other words, the variation of the orbital ordering by the change in the magnetic structure) is quite small for the whole range of JT distortion amplitudes and reaches a maximum of $\Delta \theta \approx 3^{\circ}$. In order to check whether such a small variation of the orbital mixing angle $\Delta \theta$ can cause the deviation from Heisenberg behavior which was found in Sec. III, we perform the calculations of the total energies rotating the spins from A-AFM to G-AFM ordering and using the structures with different amplitudes of JT distortion $(a=0.4$ and 1). The obtained angular dependencies of the total energy and their fittings to $f(\alpha)$ [Eq. (11)] are shown in Figs. 8(a) and 8(b). It is clearly seen that the calculated $E(\alpha)$ fit well with the Heisenberg Hamiltonian for both amplitudes of JT distortion. Therefore, one can conclude, that the variation in $\theta$ associated with the change in the magnetic order is not sufficient to explain the large deviation from the Heisenberg model which was observed in our previous calculations. It should be taken into account, however, that the energy scale is 4-6 times larger than in the case where we perform the calculations using the experimental crystal structure (Fig. 5). This is because in the latter case the exchange energy is reduced by the presence of GFO distortion. Thus, it is also possible, that the contribution from $\Delta \theta$ is not significant in comparison with the strong exchange energy within the tetragonal structure, but could be important when the orthorhombic distortion comes into play. Therefore, we are motivated to analyze next the effect of GFO distortion on the orbital and magnetic orderings.

To investigate the variation of the orbital mixing angle by GFO distortion we again construct four projected WFs. In this case, to initialize projections, we introduce a local coordinate system for each $\mathrm{MnO}_{6}$ octahedron in such a way that $x, y$, and $z$ axes are aligned as much as possible along
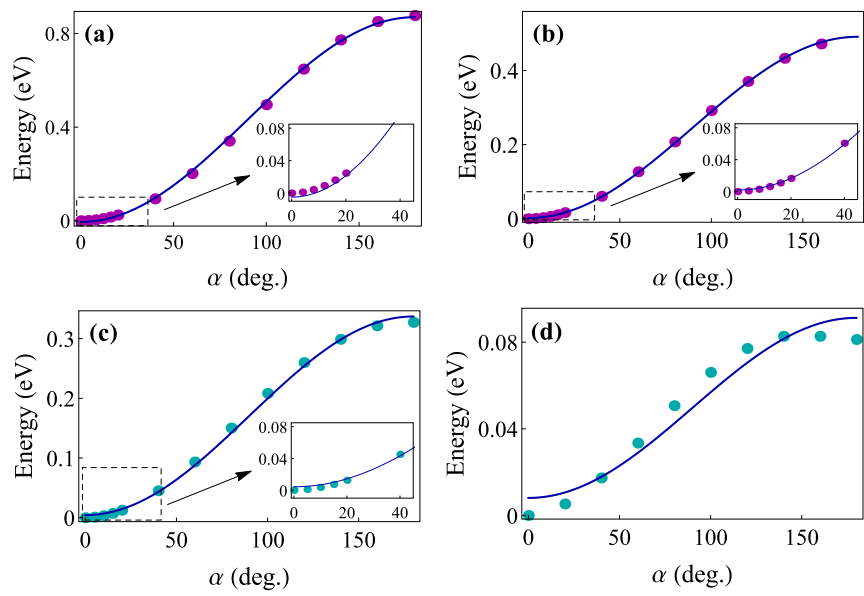

FIG. 8. (Color online) Dependence of the energy $E$ (relative to the energy of A-AFM order) on the rotation angle $\alpha$ of spins from A-AFM to G-AFM state for the structures of $\mathrm{TbMnO}_{3}$ with different amplitudes of JT and GFO distortions. Dots indicate the results of DFT calculations; lines show the fittings to the Heisenberg Hamiltonian [Eq. (11)]. (a) and (b) show the values for the structures with $40 \%$ and $100 \%$ JT distortion, respectively, without octahedral tiltings; (c) and (d) show the values for the structures with the full JT distortion and $60 \%$ and $100 \%$ GFO distortion, respectively. Plot (d) was obtained using the crystal structure which unlike the experimental one does not include the antiferroelectric displacements of $R$ cations, thus it is not identical to the one shown in Fig. 5.

the long, short, and medium Mn-O bonds, respectively. Other than that, we proceed in the same way as before: Starting from the fully JT distorted structure [Fig. 6(b)], we gradually increase the octahedral rotations to reach the experimentally observed Mn-O-Mn bond angles. The final structure is shown in Fig. 6(c); in comparison with the experimental structure, this one does not include a small antiferroelectric shift of $\mathrm{Tb}$ cations. We calculate the orbital mixing angles as a function of the amplitude of GFO distortion for A- and G-AFM orderings (Fig. 7, right part of the graph). Then we perform spin rotations from A- to G-AFM ordering with $60 \%$ and $100 \%$ GFO distorted crystal structures. Corresponding angular dependencies of the total energy are presented in Figs. 8(c) and $8(\mathrm{~d})$.

We find that increasing GFO distortion causes smaller variation of the orbital mixing angle $\left(\approx 1.5^{\circ}-3^{\circ}\right)$ in comparison with JT distortion for both magnetic orderings. Moreover, it almost does not affect $\Delta \theta$ between different types of magnetic ordering (indeed, curves for A-AFM and G-AFM stay almost parallel). However, it induces and enhances the deviation of $E(\alpha)$ from $f(\alpha)$ as shown in Figs. 8(c) and 8(d). Therefore we conclude that non-Heisenberg behavior originates from the modification of Mn-O-Mn bond angles due to the reduction of the energy of the exchange interactions between NN Mn spins, which makes weak energy contributions more significant.

In order to check whether these weak contributions are provided by $\Delta \theta$, we perform the same spin rotations for two compounds which do not contain JT active ions (therefore, do not have an orbital ordering), $\mathrm{TbCrO}_{3}\left(\mathrm{Cr}^{3+}: t_{2 g}^{3} e_{g}^{0}\right)$ and $\mathrm{TbFeO}_{3}\left(\mathrm{Fe}^{3+}: t_{2 g}^{3} e_{g}^{2}\right)$. In these calculations we use 

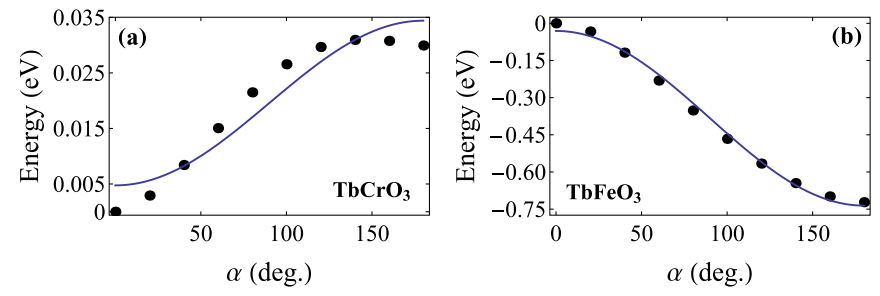

FIG. 9. (Color online) Dependence of the energy $E$ (relative to the energy of A-AFM order) on the rotation angle $\alpha$ of spins from the A-AFM to G-AFM state for compounds without orbital ordering: (a) $\mathrm{TbCrO}_{3}$ and (b) $\mathrm{TbFeO}_{3}$. Dots correspond to the results of DFT calculations; lines show the fittings to the Heisenberg Hamiltonian [Eq. (11)].

the structure of $\mathrm{TbMnO}_{3}$ with $\mathrm{Mn}^{3+}$ replaced by $\mathrm{Cr}^{3+}$ and $\mathrm{Fe}^{3+}$, respectively, which allows us to modify the strength of NN exchange interactions by changing the occupation of $d$ orbitals without any variation of the crystal structure. Indeed, in $\mathrm{TbCrO}_{3}$ magnetic couplings are mostly provided by the hopping processes between $t_{2 g}$ orbitals (mediated by oxygen $p$ states) as $e_{g}$ orbitals are empty, whereas in $\mathrm{TbFeO}_{3}$ both $t_{2 g}$ and $e_{g}$ states of one spin direction are fully occupied and participate in exchange interactions. Moreover, the $e_{g}$ orbitals in octahedral coordination have stronger overlap with $\mathrm{O} p$ states than the $t_{2 g}$ due to their geometry, and therefore provide stronger coupling. As a result, one can expect significantly larger magnitudes of $\mathrm{NN}$ exchanges for $\mathrm{TbFeO}_{3}$ than for $\mathrm{TbCrO}_{3}$.

The calculated $E(\alpha)$ are presented in Fig. 9. Both compounds exhibit deviations of $E(\alpha)$ from the cosinusoidal behavior even in the absence of an orbital ordering. Notably, the deviation is stronger for the case of $\mathrm{TbCrO}_{3}$ than for $\mathrm{TbFeO}_{3}$. We assume that in $\mathrm{TbCrO}_{3}$ the couplings which are not considered in the Heisenberg Hamiltonian are comparable in magnitude with $\mathrm{NN}$ exchanges between the $\mathrm{Mn} t_{2 g}$ states and cause a stronger deviation from cosinusoidal behavior, whereas in $\mathrm{TbFeO}_{3}$ they are not significant relative to the strong NN exchange and the Heisenberg model works sufficiently well. Thus, the observed non-Heisenberg behavior cannot be explained by the presence of the orbital ordering. Instead, it appears in the materials where the energy of the exchange couplings is reduced by the modification of $\mathrm{Mn}-\mathrm{O}-\mathrm{Mn}$ bond angles or by the occupation of the orbitals participating in the superexchange.

\section{B. Higher order exchange couplings}

Next we investigate whether exchange couplings of higher order than the usual bilinear term might be responsible for the observed deviation from the Heisenberg model. Generally speaking, the higher order exchanges as well as the bilinear coupling can be derived from a half-filled Hubbard model in the limit $t / U \ll 1$ (which is applicable for insulators),

$$
H=-t \sum_{\langle i, j\rangle, \sigma}\left(c_{i \sigma}^{\dagger} c_{j \sigma}+c_{j \sigma}^{\dagger} c_{i \sigma}\right)+U \sum_{j} \hat{n}_{j \uparrow} \hat{n}_{j \downarrow},
$$

where $t$ is a hopping parameter, $U$ describes the on-site Coulomb repulsion, $c_{j \sigma}^{\dagger}$ and $c_{j \sigma}$ are operators of creation and annihilation of electrons with spin $\sigma$ in the Wannier state $w\left(\mathbf{r}-\mathbf{R}_{j}\right)$, and $\hat{n}_{j \uparrow}=c_{j \uparrow}^{\dagger} c_{j \uparrow}$ is the occupation number operator. Second-order perturbation theory in $t$ gives the energy correction in the form of Heisenberg exchange, whereas the fourth order gives biquadratic, four-spin ring interactions and additional contributions to NNN couplings. The four-spin ring term describes the consecutive hopping processes between $\mathrm{NN}$ ions forming a four-site plaquette and has the following form [50,51]:

$$
\begin{aligned}
H_{4 s p} \propto & {\left[\left(\mathbf{S}_{i} \cdot \mathbf{S}_{j}\right)\left(\mathbf{S}_{k} \cdot \mathbf{S}_{l}\right)+\left(\mathbf{S}_{i} \cdot \mathbf{S}_{l}\right)\left(\mathbf{S}_{k} \cdot \mathbf{S}_{j}\right)\right.} \\
& \left.-\left(\mathbf{S}_{i} \cdot \mathbf{S}_{k}\right)\left(\mathbf{S}_{j} \cdot \mathbf{S}_{l}\right)\right],
\end{aligned}
$$

where $i, j, k$, and $l$ enumerate spins of the plaquette. For the Heisenberg model to be valid, all higher order terms should be negligible compared with the bilinear term. As their strength is defined by $t^{4} / U^{3}$, and that of the bilinear term by $t^{2} / U$, this should be the case in the limit of small enough $t / U$. However, several theoretical and experimental groups found that in some compounds these terms are significant. For example, it was shown that the results of a paramagnetic resonance study [52] of pairs of $\mathrm{Mn}^{2+}$ ions in $\mathrm{MgO}$ fit much better with a Hamiltonian that includes biquadratic exchange $H_{b q}$ [Eq. (6)] than with the ordinary Heisenberg Hamiltonian. Later, the significance of $H_{b q}$ was invoked to explain the establishment of the E-AFM ordering [10] as we mentioned in Sec. IIB. The four-spin ring interaction was found to be important to explain the dispersion of the magnetic excitations in $\mathrm{La}_{2} \mathrm{CuO}_{4}$ measured using inelastic neutron scattering experiments [53]. It was also shown to be significant in the spin-ladder cuprates $\mathrm{SrCu}_{2} \mathrm{O}_{3}, \mathrm{CaCu}_{2} \mathrm{O}_{3}$, and $\mathrm{Sr}_{2} \mathrm{CuO}_{4}$ using ab initio quantum chemistry embedded cluster calculations [54].

First, let us check whether the presence of the higher order terms can explain the inconsistent values of the exchange coupling constant $J_{c}$ in $\mathrm{TbMnO}_{3}$ which were obtained in Sec. III starting from different states with collinear spin alignment. We already mentioned that the addition of $H_{b q}$ cannot affect the resulting values of exchanges as the applied method considers the energy differences between states with collinear spin orientations and in these differences biquadratic terms always cancel out. Fourth-order contributions to NNN interactions, if present, are already included in the analysis as they cannot be distinguished from the bilinear NNN couplings. To introduce the terms describing the four-spin ring exchanges we have to consider the couplings between spins in the plaquettes confined in the $a b$ planes as well as from those that contain pairs of $\mathrm{Mn}$ spins from neighboring $a b$ planes. We denote the corresponding coupling constants as $G$ and $K$ [see Fig. 11(a)]. Thus, we can write the energies $E_{\uparrow \uparrow}, E_{\uparrow \downarrow}$, $E_{\downarrow \uparrow}$, and $E_{\downarrow \downarrow}$ for the 80 atom supercell of $\mathrm{TbMnO}_{3}$ in A-AFM and FM cases including four-spin interactions and put them in Eq. (9) to extract $J_{c}$. In this way we find that the in-plane ring exchanges $G$ cancel each other for both cases in the linear combinations of these energies and obtain (for $S=1$ )

$$
J_{c}(\mathrm{~A}-\mathrm{AFM}) \rightarrow J_{c}-4 K, \quad J_{c}(\mathrm{FM}) \rightarrow J_{c}+4 K .
$$

This result shows that the presence of $K$ is the most likely origin of the difference in the obtained values of $J_{c}$. Using Eq. (14) and values of $J_{c}(\mathrm{~A}-\mathrm{AFM})$ and $J_{c}(\mathrm{FM})$ which were 
obtained in Sec. III, one finds the value of $K \approx 0.6 \mathrm{meV}$ for $\mathrm{TbMnO}_{3}$.

The same can be done for the 40-atom supercell of $\mathrm{TbMnO}_{3}$ with magnetic configurations shown in Fig. 4. As before, here we find that the in-plane four-spin couplings $G$ cancel each other. The contributions to $J_{c}$ arising from the interplane ring exchanges obtained for these states are summarized in Table III. One can see the relation between these contributions and the values of $J_{c}$ which were calculated with DFT using the structures (a)-(d) and presented in the first line of Table III. Indeed, for the states (a) and (b), the interplane four-spin exchanges contribute exactly the same to $J_{c}\left(E_{4 s p}=-4 K\right)$, and the values of $J_{c}$ which we extracted using DFT for these states are very similar. States (a) and (c) as well as (c) and (d) have contributions to $J_{c}$ which differ by $4 K$. Notably, the $J_{c}$ values which we extracted for these states vary by approximately the same amount (in average $1.85 \mathrm{meV}$ ). This gives the value of $K \approx 0.5 \mathrm{meV}$, which is in agreement with the value of $K$ obtained using Eq. (14). Thus we confirm the presence of the strong four-spin interplane exchange couplings in $\mathrm{TbMnO}_{3}$ and show that the addition of these couplings to the model Hamiltonian can explain the inconsistent values of NN exchanges which were found in Sec. III.

The size of the in-plane four-spin coupling can be estimated similarly by choosing the appropriate collinear spin states and calculating energy differences for them. However, we proceed in a different way. As we already calculated the total energies of the 80 atom supercell of $\mathrm{TbMnO}_{3}$ for a large number (namely 54) of inequivalent magnetic collinear states, we can write the energies of these states using the model Hamiltonian that includes bilinear and four-spin ring couplings and construct an overdetermined system of linear equations, where the unknowns are the exchange coupling constants (bilinear ones (see Fig. 3): $J_{c}, J_{a b}, J_{a}, J_{\text {diag }}, J_{b}, J_{3 a b}$, and four-spin ones [Fig. 11(a), $G$ and $K$ ]). To build this system of equations we use only the states which are insulating and take the energy of the A-AFM state as a reference. Then we use the least mean square method to extract all coupling constants. The obtained values are presented in Table IV. We find that the in-plane four-spin coupling $G$ is negligible in comparison with the interplane one $K$. Further investigation is required to find an explanation for this observation.

Using our extracted values of the coupling constants, we calculate the expected energies of all 54 states using the considered model Hamiltonian. We plot them versus the energies of these states (referred to the energy of the A-AFM state) calculated using DFT in order to examine how well our model predicts the magnetic properties of the system (ideally, model and DFT energies should be the same). The result is
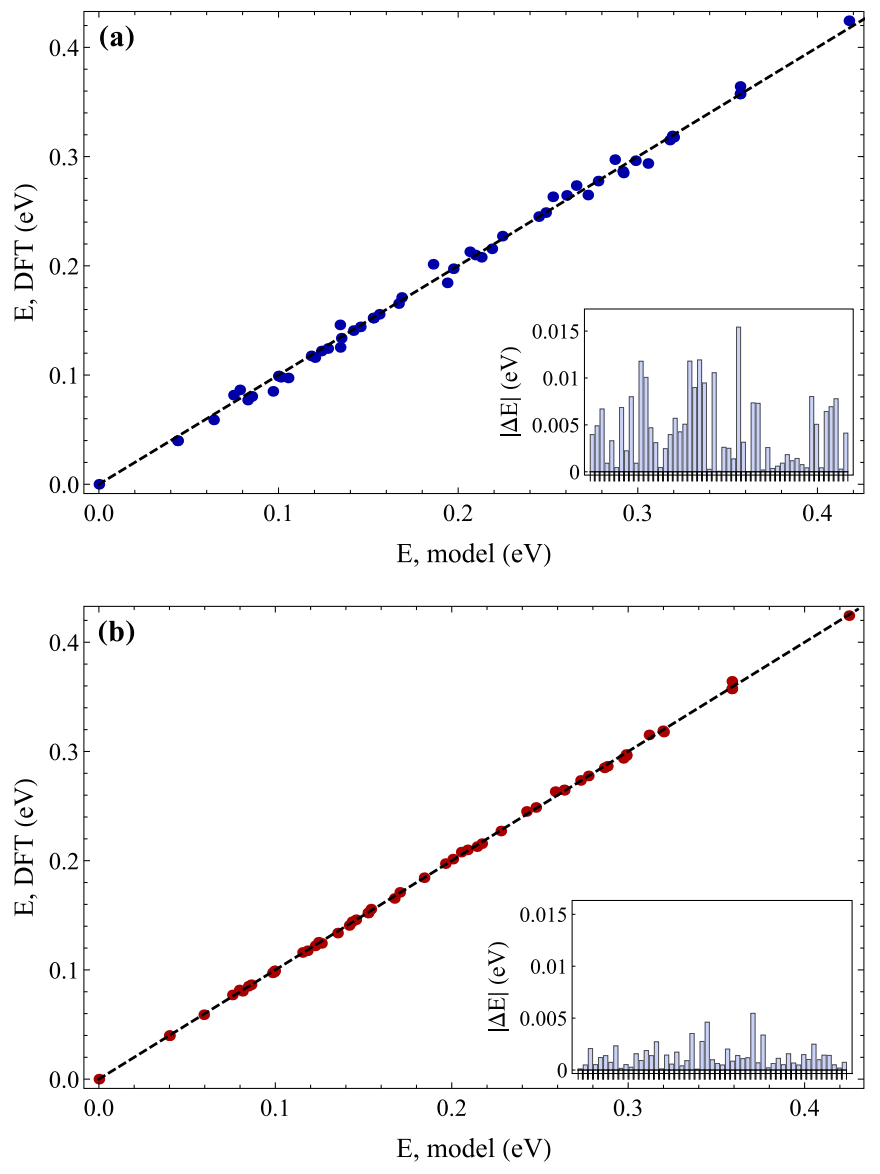

FIG. 10. (Color online) Energies of the 80 atom supercell of $\mathrm{TbMnO}_{3}$ with 54 inequivalent collinear magnetic configurations (referred to the energy of the A-AFM state) predicted by (a) the pure Heisenberg Hamiltonian and (b) the Hamiltonian, which includes bilinear and four-spin ring couplings, and plotted versus the energies of corresponding states calculated using DFT. Ideally, the model and DFT energies should be equal and points should lie on the dashed line. Insets show the deviations of the model energies from those calculated using DFT. Each bar corresponds to one considered magnetic configuration.

presented in Fig. 10(b). Similarly, we extract the coupling constants and calculate the energies of the magnetic states using the pure Heisenberg Hamiltonian (in an overdetermined system of equations, the only unknowns are the bilinear coupling constants: $J_{c}, J_{a b}, J_{a}, J_{\text {diag }}, J_{b}$, and $\left.J_{3 a b}\right)$. The model energies plotted versus the energies obtained from first-principles calculations are shown in Fig. 10(a). Moreover, we extract the deviations of the energies predicted by both

TABLE IV. Coupling constants (in meV) for bilinear $\left(J_{c}, J_{a b}, J_{a}, J_{\text {diag }}, J_{b}\right.$, and $\left.J_{3 a b}\right)$, four-spin ring $(G$ and $K)$, and biquadratic $\left(j_{c}\right.$ and $\left.j_{a b}\right)$ exchanges calculated using different values of $U$ and $J_{H}$ (in eV) for $\mathrm{TbMnO}_{3}, \mathrm{PrMnO}_{3}$, and $\mathrm{LuMnO}_{3}$.

\begin{tabular}{|c|c|c|c|c|c|c|c|c|c|c|c|c|}
\hline & $U$ & $J_{H}$ & $J_{c}$ & $J_{a b}$ & $J_{a}$ & $J_{\text {diag }}$ & $J_{b}$ & $J_{3 a b}$ & $G$ & $K$ & $j_{c}$ & $j_{a b}$ \\
\hline \multirow[t]{2}{*}{$\mathrm{TbMnO}_{3}$} & 2.0 & 0.0 & 1.22 & -6.01 & -0.47 & 0.31 & 0.65 & 1.21 & -0.05 & 0.50 & -0.81 & -2.29 \\
\hline & 3.0 & 1.0 & 4.78 & 0.02 & -0.29 & 0.22 & 0.68 & 0.96 & 0.07 & 0.28 & -0.62 & -1.44 \\
\hline $\mathrm{PrMnO}_{3}$ & 1.0 & 0.0 & 1.79 & -14.16 & -0.48 & 0.88 & 0.26 & 3.25 & -0.07 & 0.80 & -2.61 & -2.95 \\
\hline $\mathrm{TbMnO}_{3}$ & 1.0 & 0.0 & 4.26 & -3.86 & -0.37 & 0.58 & 0.85 & 1.77 & -0.02 & 0.77 & -0.47 & -2.09 \\
\hline $\mathrm{LuMnO}_{3}$ & 1.0 & 0.0 & 3.76 & -0.48 & -0.55 & 0.53 & 0.93 & 1.75 & 0.15 & 0.66 & -0.37 & -2.29 \\
\hline
\end{tabular}


Hamiltonians from their values obtained with DFT for each considered magnetic state. These deviations are summarized in the bar charts shown in the insets in Fig. 10. One can see that the Hamiltonian which includes both bilinear and four-spin terms gives much better agreement with the results of DFT calculations than the pure Heisenberg Hamiltonian. We repeat this analysis also for the Hamiltonian which involves six bilinear exchange couplings and only interplane four-spin ring coupling $K$, as $G$ was found to be negligible. The extracted coupling constants as well as the deviations between model and DFT energies remain almost the same as those which were obtained using the full Hamiltonian (which includes also $G)$. This means that the addition of just one parameter $K$ into the model Hamiltonian can already significantly improve the description of the magnetism in the considered compound.

The effect of the higher order exchange interactions in $\mathrm{TbMnO}_{3}$ can also be examined using noncollinear calculations. The simplest approach is to map the angular dependence of the total energy, obtained in Sec. III from the spin rotations from A-AFM to G-AFM orderings, to the Hamiltonian which includes bilinear, biquadratic, and four-spin ring interactions by fitting to the function,

$$
g(\alpha)=A_{2}+B_{2} \cos (\alpha)+C_{2} \cos ^{2}(\alpha) .
$$

The result is shown in Fig. 5 and clearly demonstrates that the introduction of the higher order couplings into the model Hamiltonian greatly improves the fitting. The strengths of these couplings are determined by the fitting parameter $C_{2}$. Note that this term includes the contributions from four-spin ring exchanges as well as from the in-plane biquadratic couplings and that these terms cannot be separated. Similar behavior of $E(\alpha)$ was found in Ref. [36] for hexagonal $\mathrm{YMnO}_{3}$ using $a b$ initio calculations within the LDA $+\mathrm{U}$ approximation, where it was discussed only in terms of bilinear and biquadratic exchanges.

To complete the analysis of the full model Hamiltonian we need to estimate the coupling constants which define the biquadratic exchanges in $\mathrm{TbMnO}_{3}$. For this we only take into account the biquadratic interactions between the nearest neighbors in $a b$ planes and along the $c$ axis and denote the corresponding coupling constants as $j_{a b}$ and $j_{c}$ (see Fig. 11). The problem can be simplified if we eliminate the contribution from four-spin interplane ring exchanges by considering the magnetic states which set to zero at least one scalar product in each of the three terms in Eq. (13). This can be achieved by setting three Mn spins in the unit cell perpendicular to each other as shown in Fig. 11. By rotation of the remaining spin the angular dependence of the total energy can be obtained and the coupling constants can be found from the corresponding fittings. To extract $j_{a b}$ we start from the magnetic state shown in Fig. 11(a) and rotate spin 4 by an angle $\alpha$ from $0^{\circ}$ to $180^{\circ}$ in the $a c$ plane. The energy of this system can be written as follows:

$$
\begin{aligned}
E(\alpha)= & E+4 J_{a b} \cos (\alpha)+8 J_{\text {diag }} \sin (\alpha) \\
& +4 j_{a b} \cos ^{2}(\alpha)+8 G \cos ^{2}(\alpha),
\end{aligned}
$$

where the third term is given by spins 1 and 4 and all other terms by spins 3 and $4(S=1)$ and $E$ includes the exchange couplings which are constant at every $\alpha$ for the considered

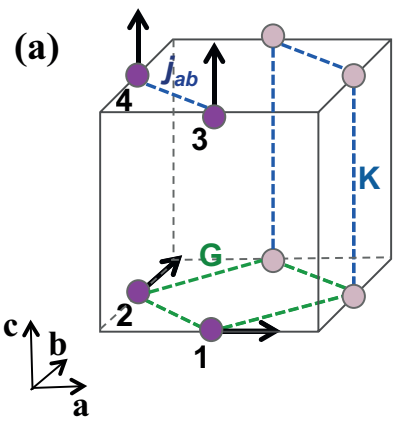

(b)

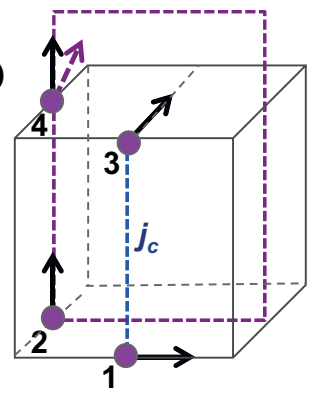

FIG. 11. (Color online) Magnetic orderings which are used to extract the biquadratic exchange interactions: (a) $j_{a b}$ in the $a b$ planes ( $G$ and $K$ indicate four-spin ring exchange couplings in plaquettes of Mn spins confined in the $a b$ planes and those containing pairs of spins from neighboring $a b$ planes, respectively); (b) $j_{c}$ along the $c$ axis (violet dashed rectangle indicates a rotation plane of spin 4).

magnetic states and other nonmagnetic interactions. The coupling constants can be extracted by fitting to the function:

$$
\begin{aligned}
f(\alpha)= & A_{1}+4 J_{a b} \cos (\alpha)+8 J_{\text {diag }} \sin (\alpha) \\
& +\left(D_{1}+8 G\right) \cos ^{2}(\alpha),
\end{aligned}
$$

where we set the values of $J_{a b}, J_{\text {diag }}$, and $G$ to those which were extracted in the collinear calculations and presented in the first line of Table IV. $D_{1} / 4$ defines $j_{a b}=-2.29 \mathrm{meV}$. We proceed in a similar way to extract the coupling constant $j_{c}$. Starting from the magnetic state presented in Fig. 11(b) and rotating spin 4 by $\alpha$ from $0^{\circ}$ to $180^{\circ}$ in the $a c$ plane, we obtain $E(\alpha)$. One can see that in the considered magnetic state neither in-plane nor interplane four-spin ring couplings contribute to $E(\alpha)$ and fitting to

$$
g(\alpha)=A_{2}+2 J_{c} \cos (\alpha)+8 J_{\text {diag }} \sin (\alpha)+D_{2} \cos ^{2}(\alpha),
$$

gives $j_{c}=D_{2} / 2=-0.81 \mathrm{meV}$.

Thus we demonstrate that the higher order exchange interactions are significant in $\mathrm{TbMnO}_{3}$ (especially the four-spin ring interplane coupling $K$ and biquadratic in-plane coupling $j_{a b}$ ) and have to be included in the model Hamiltonian to properly describe the magnetic properties of this material.

\section{V. $U$ AND $J_{H}$ DEPENDENCE OF EXCHANGE COUPLINGS}

In general, microscopic exchange interactions calculated within the DFT $+\mathrm{U}$ approach are sensitive to the choice of the on-site Coulomb repulsion $U$ and the on-site exchange interaction $J_{H}$. Indeed, as was shown in Ref. [55] for the case of $\mathrm{LaMnO}_{3}$, the effective $e_{g}$ hopping amplitudes depend significantly on $U$. In addition $J_{H}$ has been shown to have large effects on the magnetic ground state of noncollinear magnets [56]. Therefore, it is important to investigate how the relative strength of bilinear and higher order terms varies with these parameters.

First, we analyze the effect of $U$ by performing spin rotations from A-AFM to G-AFM states (see Sec. II C 2) within the 20-atom unit cell of $\mathrm{TbMnO}_{3}$ and setting $U=4 \mathrm{eV}$ and $8 \mathrm{eV}$ (with $J_{H}=0 \mathrm{eV}$ ). We map the obtained $E(\alpha)$ (relative to the energy of the A-AFM state) onto both the pure 

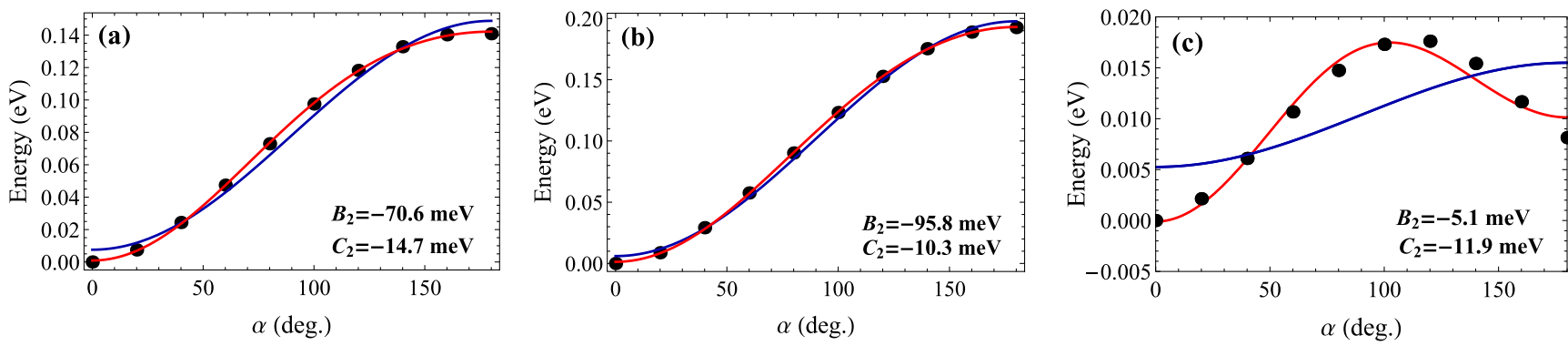

FIG. 12. (Color online) Dependencies of the energy $E$ (relative to the energy of A-AFM order) of $\mathrm{TbMnO}_{3}$ on the rotation angle $\alpha$ of spins from A-AFM to G-AFM state calculated using (a) $U=4 \mathrm{eV}$ and $J_{H}=0 \mathrm{eV}$; (b) $U=8 \mathrm{eV}$ and $J_{H}=0 \mathrm{eV}$; (c) $U=3 \mathrm{eV}$ and $J_{H}=1 \mathrm{eV}$. The results of DFT calculations are shown by dots. The blue line indicates the fitting to the Heisenberg model [Eq. (11)] and the red line-fitting to a Hamiltonian which includes bilinear and higher order exchange couplings [Eq. (15)]. $B_{2}$ and $C_{2}$ are the fitting parameters of Eq. (15), which define sizes of the bilinear and higher order terms, respectively.

Heisenberg Hamiltonian and then on the Hamiltonian that also includes biquadratic and four-spin ring terms, by fitting to $f(\alpha)$ [Eq. (11)] and $g(\alpha)$ [Eq. (15)], respectively. The size of bilinear and higher order terms are defined by fitting parameters $B_{2}$ and $C_{2}$ of Eq. (15). The obtained curves and corresponding fitting parameters are summarized in Figs. 12(a) and 12(b). One can see that for both values of $U$, the addition of biquadratic and four-spin ring terms to the model Hamiltonian significantly improves the fitting of DFT energies. Although the effect of these terms seems less pronounced for the case of $U=8 \mathrm{eV}$, the reason for this is the drastic increase of the bilinear couplings with respect to the higher order contributions, which are less affected by increasing $U$.

Next, to investigate the effect of $J_{H}$ we set $U=3 \mathrm{eV}$ and $J_{H}=1 \mathrm{eV}$ corresponding to the same value of $U_{\text {eff }}=U-$ $J_{H}=2 \mathrm{eV}$, which was used in all calculations described in the previous sections. We extract again $E(\alpha)$ for $\mathrm{TbMnO}_{3}$ by rotating spins from A-AFM to G-AFM order. The obtained angular dependence of the energy of the system (relative to the energy of A-AFM order) and its fits to $f(\alpha)$ [Eq. (11)] and $g(\alpha)$ [Eq. (15)] are presented in Fig. 12(c). We find that the addition of $J_{H}$ in the calculations strongly changes the energy of magnetic interactions. Indeed, the energy scale is reduced by more than a factor of 5 relative to the case of $U=2 \mathrm{eV}$ and $J_{H}=0 \mathrm{eV}$ (see Fig. 5). By comparing the fitting parameters $B_{2}$ and $C_{2}$ for these two cases one can see that the strength of the bilinear couplings is sharply reduced by the addition of $J_{H}$. Moreover, it makes the effect of higher order terms even more significant as their magnitude is now larger than the one of the bilinear terms.

To understand which exchanges have the strongest $J_{H}$ dependence we extract all coupling constants separately for the full model Hamiltonian (which includes bilinear, biquadratic, and four-spin ring terms). We calculate the total energies of 45 inequivalent collinear spin configurations using the 80 atom supercell of $\mathrm{TbMnO}_{3}$ and setting $U=3 \mathrm{eV}$ and $J_{H}=1 \mathrm{eV}$. Then we construct an overdetermined system of equations with exchange couplings $J_{c}, J_{a b}, J_{a}, J_{\text {diag }}, J_{b}, J_{3 a b}, G$ and $K$ as variables, and with the E-AFM energy as the reference. Using the least mean square method we obtain all these couplings. After that we extract biquadratic NN in-plane $j_{a b}$ and interplane $j_{c}$ couplings by the same procedure as was described in Sec. IV B. All obtained values of exchanges are presented in Table IV. One can see that $J_{c}$ and especially $J_{a b}$ drastically change on the addition of $J_{H}$ and the latter even changes sign, while other couplings are affected much less by $J_{H}$. In this case, $J_{b}$ becomes strong enough in comparison to $J_{a b}$ to provide a spiral state according to Eq. (5). Moreover, this result confirms that all higher order couplings $K, j_{c}$, and $j_{a b}$ become more significant for $J_{H}=1 \mathrm{eV}$ as they are now even stronger than the bilinear $\mathrm{NN}$ in-plane term.

Thus we find that both $U$ and $J_{H}$ have a dramatic effect on the values of the calculated exchange couplings (especially, the bilinear $\mathrm{NN}$ ones) and have to be accurately chosen to relate the results of DFT calculations to experimental data. Understanding of this behavior of the exchange couplings and determination of the values of $U$ and $J_{H}$ that give the best agreement with the experiment, requires further investigations, which go beyond the scope of the present paper. Nevertheless, we can definitely say that the higher order terms are significant and have to be considered for all reasonable values of $U$ and $J_{H}$.

\section{EXCHANGE INTERACTIONS IN OTHER O- $\mathrm{RMNO}_{3}$}

Finally, in this section we investigate the evolution of the exchange couplings in o- $R \mathrm{MnO}_{3}$ with increasing GFO distortion due to decrease in the radius of the $R$ cation. For this purpose we consider $\mathrm{PrMnO}_{3}$ and $\mathrm{LuMnO}_{3}$, which have among the largest and the smallest $R$ radii in the series of o- $R \mathrm{MnO}_{3}$, respectively. We set $U=1 \mathrm{eV}$ (and $J_{H}=0 \mathrm{eV}$ ) for Mn $d$ states, which gives the correct magnetic ground state for both considered systems. We calculate the total energies of these systems within the 80 atom supercells (experimental unit cells $[41,42]$ are duplicated along $a$ and $b$ directions) for 34 and 32 inequivalent collinear magnetic states, respectively. By writing the expressions for the energies of these magnetic states using the model Hamiltonian that includes bilinear and four-spin ring exchange interactions, we obtain overdetermined systems of equations with respect to six bilinear and two four-spin ring couplings for each compound. In both cases the lowest energy state was taken as the reference (the A-AFM state for $\mathrm{PrMnO}_{3}$ and the E-AFM state for $\mathrm{LuMnO}_{3}$ ). We solve these systems of equations using the least mean square method and find the values of all coupling constants (Table IV). For comparison, we calculate the exchange couplings in a similar way for $\mathrm{TbMnO}_{3}$ for the considered value of $U$. From Table IV one can see that the increasing GFO distortion has the strongest effect on the in-plane NN coupling $J_{a b}$ which changes by more than one 

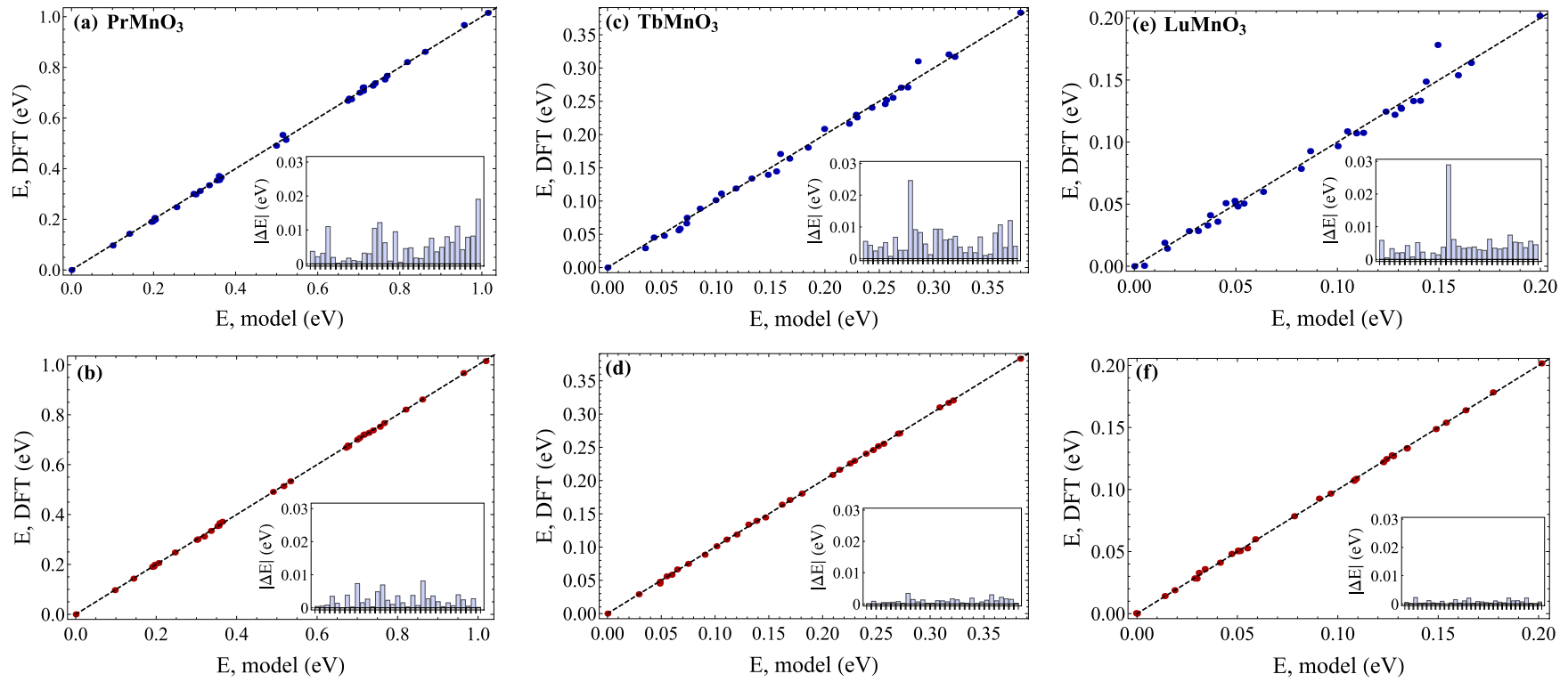

FIG. 13. (Color online) Energies of the 80 atom supercells of $\mathrm{PrMnO}_{3}$ (a) and (b), $\mathrm{TbMnO}_{3}$ (c) and (d), and $\mathrm{LuMnO}_{3}$ (e) and (f) with more than 30 inequivalent magnetic configurations (referred to that of the lowest-energy state) predicted by the pure Heisenberg Hamiltonian (blue dots) and the Hamiltonian which includes bilinear and four-spin ring exchanges (red dots) and plotted versus the energies of corresponding states calculated using DFT.

order of magnitude from Pr to Lu. It also enhances $J_{b}$ as we expected (see Sec. II B). However, the behavior of the other coupling constants (particularly the absence of a trend in the variation of $J_{c}$ and $J_{a}$, and the strong change in some coupling constants and weak change in others) with the variation of $\mathrm{Mn}-\mathrm{O}-\mathrm{Mn}$ bond angles still requires further analysis.

As the next step, we use the extracted values of the exchange couplings to calculate the energies of all considered magnetic states within this model Hamiltonian and plot them versus the energies of these states which we calculate using DFT [see Figs. 13(b), 13(d), and 13(f)]. For comparison, we extract similarly the coupling constants using the pure Heisenberg Hamiltonian for each compound, then calculate the energies of all states, predicted by this Hamiltonian, and plot them versus the DFT energies of these states [Figs. 13(a), 13(c), and 13(e)]. The insets in all resulting graphs show the deviations of the model energy from the DFT energy for each considered state. From Fig. 13 we find that the model Hamiltonian which includes both the bilinear and four-spin ring exchanges gives much better agreement with the results of the first-principles calculations. One can conclude that the Heisenberg model works relatively well for $\mathrm{PrMnO}_{3}$, since the model and DFT energies almost coincide in Fig. 13(a). However, if one compares the $\Delta E$ values which were obtained for the bilinear-only case [see insets in Figs. 13(a), 13(c), and 13(e)], one can see that they are similar and even larger than those of $\mathrm{TbMnO}_{3}$ and $\mathrm{LuMnO}_{3}$, but small relative to the energy scale of the bilinear couplings, in particular, $J_{a b}$ (see Table IV). When GFO distortion increases and $J_{a b}$ drops (as in $\mathrm{TbMnO}_{3}$ and $\mathrm{LuMnO}_{3}$ ), the $\Delta E$ due to the non-Heisenberg terms become significant. However, when the four-spin ring couplings are added in the model Hamiltonian [Figs. 13(b), 13(d), and 13(f)], the $\Delta E$ values reduce drastically.

Finally, we extract the biquadratic couplings $j_{c}$ and $j_{a b}$ for $\mathrm{PrMnO}_{3}, \mathrm{TbMnO}_{3}$, and $\mathrm{LuMnO}_{3}$ (see Table IV) applying the method which was described in detail at the end of Sec. IV B. For all compounds we obtain strong negative in-plane biquadratic couplings $j_{a b}$, which favor collinear alignment of spins within the $a b$ planes and can drive an evolution of a magnetic order from a spiral to an E-AFM state for systems with large GFO distortions. This confirms the finding of Ref. [10], where the biquadratic exchange interaction was claimed to be important in the establishment of the E-AFM order. The in-plane coupling $j_{c}$ is found to be much more affected by GFO distortion than $j_{a b}$. Again, the origin of this behavior still has to be clarified.

Thus we show that the Heisenberg Hamiltonian cannot accurately predict the magnetic properties of $0-R \mathrm{MnO}_{3}$ with large GFO distortions. In these materials the bilinear couplings become comparable in magnitude with the biquadratic and four-spin ring interactions and it is essential to include the latter two into the model Hamiltonian for proper analysis of the magnetism.

\section{SUMMARY AND CONCLUSIONS}

In summary, we investigated the microscopic exchange

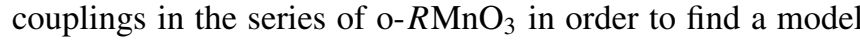
Hamiltonian (excluding effects due to spin-orbit coupling like SIA and DMI) which can properly describe the magnetism in these materials. The work was motivated by the inconsistencies in the results obtained in several theoretical studies when the exchange couplings in o- $R \mathrm{MnO}_{3}$ was mapped onto the Heisenberg Hamiltonian as well as by the absence of agreement on the origin of the E-AFM order in o- $R \mathrm{MnO}_{3}$ with small $R$ cations $(R=$ Ho $\ldots \mathrm{Lu})$. We started our analysis from the most studied multiferroic orthorhombic manganite, $\mathrm{TbMnO}_{3}$, and estimated the exchange couplings with several approaches (collinear and noncollinear) using DFT. We observed a clear deviation from the behavior predicted by the Heisenberg 
model. In the next step we explored whether the observed non-Heisenberg behavior originates from the presence of the orbital ordering in $\mathrm{TbMnO}_{3}$ and its coupling with the Mn spins. To check this, we analyzed the changes in the orbital mixing angle with structural distortions (Jahn-Teller and GFO) and with variation of the magnetic ordering using the Wannier function representation. We found that the orbital mixing angle indeed can be affected by the magnetic order, however, we showed that this variation is quite small and is almost unchanged by the structural distortions. In turn, we found that the deviation from Heisenberg behavior does not appear when the amplitude of JT distortion is varied. It appears only with increasing GFO distortion, which decreases the energy of the NN exchange interactions and makes the weak energy contributions more important. We demonstrated, however, that these weak contributions do not originate from the variation of the orbital ordering. Indeed, compounds which do not have an orbital degree of freedom (such as $\mathrm{TbCrO}_{3}$ and $\mathrm{TbFeO}_{3}$ ) also exhibit a deviation from the energy behavior predicted by the Heisenberg model. Finally, we investigated the effects of exchange couplings of higher order than the ordinary bilinear exchange (biquadratic and four-spin ring interactions), which are usually neglected. We demonstrated that the higher order contributions are significant (especially interplane four-spin ring exchange $K$ and biquadratic inplane coupling $j_{a b}$ ) and can be comparable with the bilinear exchanges for $\mathrm{O}-\mathrm{RMnO} \mathrm{M}_{3}$ with small radii of $R$ cations. We showed that the inconsistent values of the exchange couplings which were obtained from the collinear calculations within the Heisenberg model (Sec. III) can be explained only by addition of the four-spin ring couplings into the model Hamiltonian. Moreover, we proved that such a model Hamiltonian predicts the DFT energies of o- $R \mathrm{MnO}_{3}$ with much higher accuracy than the pure Heisenberg Hamiltonian independently of the values of $U$ and $J_{H}$, which are used in the calculations. The finding of the strong negative in-plane biquadratic exchange interaction $j_{a b}$, which favors a collinear spin alignment within the $a b$ planes, is in agreement with the suggestion of Refs. [10,28] that $H_{b q}$ is crucial in the establishment of the E-AFM state.

\section{ACKNOWLEDGMENT}

This work was supported by ERC Advanced Grant program (No. 291151), and by ETH Zürich.
[1] N. A. Hill, J. Phys. Chem. B 104, 6694 (2000).

[2] N. A. Spaldin and M. Fiebig, Science 309, 391 (2005).

[3] S.-W. Cheong and M. Mostovoy, Nature Materials 6, 13 (2007).

[4] T. Kimura, T. Goto, H. Shintani, K. Ishuzaka, T. Arima, and Y. Tokura, Nature (London) 426, 55 (2003).

[5] B. Lorenz, Y.-Q. Wang, and C.-W. Chu, Phys. Rev. B 76, 104405 (2007).

[6] E. O. Wollan and W. C. Koehler, Phys. Rev. 100, 545 (1955).

[7] J.-S. Zhou and J. B. Goodenough, Phys. Rev. Lett. 96, 247202 (2006).

[8] T. Kimura, S. Ishihara, H. Shintani, T. Arima, K. Takahashi, K. Ishizaka, and Y. Tokura, Phys. Rev. B 68, 060403(R) (2003).

[9] M. Mochizuki and N. Furukawa, Phys. Rev. B 80, 134416 (2009).

[10] T. A. Kaplan, Phys. Rev. B 80, 012407 (2009).

[11] T. A. Kaplan and S. D. Mahanti, arXiv:0904.1739.

[12] J. A. Alonso, M. J. Martínez-Lope, M. T. Casais, and M. T. Fernández-Díaz, Inorg. Chem. 39, 917 (2000).

[13] C. Ederer, C. Lin, and A. J. Millis, Phys. Rev. B 76, 155105 (2007).

[14] R. Kováčik and C. Ederer, Phys. Rev. B 81, 245108 (2010).

[15] J. Kanamori, Journal of Applied Physics 31, S14 (1960).

[16] P. M. Woodward, Acta Cryst. B53, 32 (1997).

[17] N. A. Benedek and C. J. Fennie, The Journal of Physical Chemistry C 117, 13339 (2013).

[18] H. A. Jahn and E. Teller, Proc. R. Soc. Lond. A 161, 220 (1937).

[19] K. I. Kugel' and D. I. Khomskiǔ, Zh. Eksp. Teor. Fiz. 64, 1429 (1973) [Sov. Phys.-JETP 37, 725 (1973)].

[20] J. H. Van Vleck, The Journal of Chemical Physics 7, 72 (1939).

[21] D. I. Khomskii, Physica Scripta 72, CC8 (2005).

[22] J. van den Brink, New Journal of Physics 6, 201 (2004).

[23] J. H. Lee, K. T. Delaney, E. Bousquet, N. A. Spaldin, and K. M. Rabe, Phys. Rev. B 88, 174426 (2013).
[24] J. B. Goodenough, Magnetism and Chemical Bond (Interscience Publishers, Hoboken, 1963).

[25] J. B. Goodenough, Phys. Rev. 100, 564 (1955).

[26] J. Kanamori, J. Phys. Chem. Solids 10, 87 (1959).

[27] Note that the factor $1 / 2$ appears in Eq. (5) instead of the factor $1 / 4$ for the case of an infinite spin chain with competing nearestand next-nearest-neighbor couplings as we take into account the number of equivalent bonds with exchange couplings $J_{b}$ and $J_{a b}$, which are equal to 2 and 4 , respectively.

[28] L. X. Hayden, T. A. Kaplan, and S. D. Mahanti, Phys. Rev. Lett. 105, 047203 (2010).

[29] I. Solovyev, J. Phys. Soc. Jpn. 78, 054710 (2009).

[30] K. Yamauchi, F. Freimuth, S. Blügel, and S. Picozzi, Phys. Rev. B 78, 014403 (2008).

[31] R. A. Evarestov, E. A. Kotomin, Yu. A. Mastrikov, D. Gryaznov, E. Heifets, and J. Maier, Phys. Rev. B 72, 214411 (2005).

[32] P. Hohenberg and W. Kohn, Phys. Rev. 136, B864 (1964).

[33] W. Kohn and L. J. Sham, Phys. Rev. 140, A1133 (1965).

[34] H. J. Xiang, E. J. Kan, S.-H. Wei, M. H. Whangbo, and X. G. Gong, Phys. Rev. B 84, 224429 (2011).

[35] M. H. Whangbo, D. Dai, and H. J. Koo, Solid State Sciences 7, 827 (2005).

[36] P. Novák, I. Chaplygin, G. Seifert, S. Gemming, and R. Laskowski, Computational Materials Science 44, 79 (2008).

[37] G. Kresse and J. Furthmüller, Phys. Rev. B 54, 11169 (1996).

[38] J. P. Perdew, K. Burke, and M. Ernzerhof, Phys. Rev. Lett. 77, 3865 (1996).

[39] A. I. Liechtenstein, V. I. Anisimov, and J. Zaanen, Phys. Rev. B 52, R5467 (1995).

[40] S. L. Dudarev, G. A. Botton, S. Y. Savrasov, C. J. Humphreys, and A. P. Sutton, Phys. Rev. B 57, 1505 (1998).

[41] H. Okamoto, N. Imamura, B. C. Hauback, M. Karppinen, H. Yamauchi, and H. Fjellvag, Solid State Comm. 146, 152 (2008). 
[42] U. D. Wdowik, B. Ouladdiaf, and T. Chatterji, J. Phys.: Condens. Matter 23, 245402 (2011).

[43] W. Ku, H. Rosner, W. E. Pickett, and R. T. Scalettar, Phys. Rev. Lett. 89, 167204 (2002).

[44] A. A. Mostofi, J. R. Yates, Y.-S. Lee, I. Souza, D. Vanderbilt, and N. Marzari, Comput. Phys. Commun. 178, 685 (2008).

[45] C. Franchini, R. Kováčik, M. Marsman, S. Sathyanarayana Murthy, J. He, C. Ederer, and G. Kresse, J. Phys.: Condens. Matter 24, 235602 (2012).

[46] H. J. Xiang, S.-H. Wei, M.-H. Whangbo, and J. L. F. Da Silva, Phys. Rev. Lett. 101, 037209 (2008).

[47] A. Malashevich and D. Vanderbilt, Phys. Rev. Lett. 101, 037210 (2008).

[48] N. Marzari, A. A. Mostofi, J. R. Yates, I. Souza, and D. Vanderbilt, Reviews of Modern Physics 84, 1419 (2012).
[49] W.-G. Yin, D. Volja, and W. Ku, Phys. Rev. Lett. 96, 116405 (2006).

[50] P. Fazekas, Lecture Notes on Electron Correlation and Magnetism (World Scientific Publishing, Hoboken, 1999).

[51] M. Takahashi, J. Phys. C: Solid State Phys. 10, 1289 (1976).

[52] E. A. Harris and J. Owen, Phys. Rev. Lett. 11, 9 (2011).

[53] R. Coldea, S. M. Hayden, G. Aeppli, T. G. Perring, C. D. Frost, T. E. Mason, S. W. Cheong, and Z. Fisk, Phys. Rev. Lett. 86, 5377 (2001).

[54] C. J. Calzado, C. de Graaf, E. Bordas, R. Caballol, and J.-P. Malrieu, Phys. Rev. B 67, 132409 (2003).

[55] R. Kováčik and C. Ederer, Phys. Rev. B 84, 075118 (2011).

[56] E. Bousquet and N. Spaldin, Phys. Rev. B 82, 220402 (2010). 